\title{
A multi-centre, pragmatic, three-arm, individually randomised, non-inferiority, open trial to compare Immediate Oral, Immediate Topical or Delayed Oral Antibiotics for Acute Otitis Media with Discharge in children: The Runny Ear Study (REST): Study protocol.
}

\section{Kathryn Curtis}

University of Bristol Faculty of Health Sciences

Michael Moore

University of Southampton Faculty of Medicine

Christie Cabral

University of Bristol Faculty of Health Sciences

Vasa Curcin

King's College London

Jeremy Horwood

University of Bristol Faculty of Health Sciences

Richard Morris

University of Bristol Faculty of Health Sciences

Vibhore Prasad

Kings London

Anne Schilder

UCL Ear Institute

Nicholas Turner

University of Bristol Faculty of Health Sciences

Scott Wilkes

University of Sunderland, School Of Medicine

Alastair Hay

University of Bristol Faculty of Health Sciences

Jodi Taylor ( $\boldsymbol{\nabla}$ j.taylor@bristol.ac.uk)

University of Bristol School of Social and Community Medicine 
Keywords: Acute otitis media, primary care, antibiotics, paediatrics

Posted Date: April 15th, 2020

DOI: https://doi.org/10.21203/rs.3.rs-19914/v1

License: (c) (1) This work is licensed under a Creative Commons Attribution 4.0 International License. Read Full License 


\section{Abstract}

Background Acute otitis media (AOM) is a common painful infection in children, with around 2.8 million cases presenting to primary care in England and Wales annually. Nearly all children who present to their general practitioner (GP) with $\mathrm{AOM}$ or $\mathrm{AOM}$ with discharge (AOMd) are treated with oral antibiotics. These can cause side effects; contribute to the growing problem of antimicrobial resistance, and more rarely, allergic reactions. Alternative treatments, such as an antibiotic eardrops or "delayed" oral antibiotics, could be at least as effective and safe as immediate oral antibiotics for children with AOMd. Methods REST is a pragmatic, three-arm, individually randomised, non-inferiority trial being conducted in $50 \mathrm{GP}$ practices across the UK. The study aims to recruit 399 children aged $(\geq 12 \mathrm{~m}$ and $<16 y r s)$ presenting to their GP with AOMd. Children will be randomised to one of three arms: immediate ciprofloxacin $0.3 \%$ eardrops; delayed oral amoxicillin (clarithromycin if penicillin allergic); or immediate oral amoxicillin (clarithromycin). Recruitment, including eligibility screening, randomisation and data collection, are conducted using the innovative, TRANSFoRm electronic trial management platform. Integrated within the primary care electronic medical records it provides automatic eligibility checking, part-filling of e-CRFs, study workflow management and routine NHS follow up data collection. The primary outcome is time to resolution of all significant symptoms and will be collected by the parent using a Symptom Recovery Questionnaire (SRQ). Secondary outcomes, including cost-effectiveness, duration of moderately bad or worse symptoms and repeat AOMd episodes, will be collected at day 14 and at 3 months. Discussion It is unclear whether prescribing oral antibiotics to children with AoMd results in a reduction in symptoms or a shorter duration of illness. The REST trial will allow us to compare the non-inferiority of: immediate topical ciprofloxacin ear drops, or delayed oral amoxicillin (clarithromycin) against immediate oral amoxicillin (clarithromycin). We aim to recruit 399 patients from 175 practices in the UK. Using the Transform software to randomise participants to the trial will enable recruitment for a relatively uncommon condition.

\section{Trial Registration:}

Name of Registry: ISCRTN

Registration Number: ISRCTN12873692

Date of Registration: 24th April 2018

http://www.isrctn.com/ISRCTN12873692

Name of Registry: EudraCT

Registration Number: 2017-003635-10

Date of Registration: $6^{\mathrm{TH}}$ September 2017 


\section{Background And Rationale}

Acute otitis media (AOM) is important to children, parents and the NHS. Firstly, the infection causes pain and distress to the child, disrupting sleep and family routines. In around $15 \%$ of cases, a rise in middle ear pressure and/or inflammation weakening the tympanic membrane results in it bursting, discharging pus from the middle ear as a discharge (otorrhoea) ${ }^{1}$. Children with AOM and discharge (AOMd) have similar levels of pain and are more unwell at presentation than children with AOM. ${ }^{34}$ Moreover, children with AOMd have a worse prognosis, and higher rates of pain at one week, as reported by parents (carers), repeat $\mathrm{AOM}$ episodes and hearing problems at 3 months $^{3}$. Estimates of parental costs (travel, over the counter (OTC) medicines and lost earnings) vary ${ }^{5-7}$, with even the lowest suggesting $£ 4 \mathrm{M}$ in England and Wales per annum.

Over $90 \%$ of UK parents attend primary care health services for each episode of $\mathrm{AOMd}^{8}$, with over 150,000 GP consultations for AOMd in England and Wales per annum at a cost to the NHS of over $£ 3 M^{5}$ 6 , which is more than for any other common symptom of acute infection.

More children with AOM and AOMd receive an oral antibiotic in the $\mathrm{UK}^{9}$ and $\mathrm{US}^{10}$ than for any other respiratory infection, with three-quarters of GPs prescribing oral antibiotics to at least $80 \%$ of children diagnosed as such ${ }^{11} 12$. Our 2015 audit including $33 \mathrm{GP}$ practices and 56,251 children, confirmed immediate oral antibiotics is usual care for AOMd: $88 \%$ were given oral antibiotics of which $97 \%$ were immediate.

There is strong evidence that children with AOMd benefit from immediate oral antibiotics. The number needed to treat with antibiotics is three to reduce the proportion of children with pain and/or fever at 3 to 7 days compared with placebo/no treatment ${ }^{4}$. The National Institute for Health and Care Excellence (NICE) therefore recommends immediate antibiotics should be considered ${ }^{13}$. Oral antibiotics do however also cause side effects, are associated with subsequent eczema and hay fever ${ }^{14}$ and with population ${ }^{15}$ and patient ${ }^{16}$ level antimicrobial resistance.

In response to the UK's Antimicrobial Resistance Action Plan calls for research to preserve antibiotic effects ${ }^{17}$ we have designed a trial of topical antibiotics in children with AOMd, aiming to reduce the use of systemic antibiotics. Perforation of the tympanic membrane provides a port of entry into the middle ear for antibiotic drops instilled in the ear canal. In children with ventilation tubes ('grommets'), it has been shown that topical antibiotics can reach the infected middle ear despite purulent discharge ${ }^{18}$, and that compared with oral antibiotics, they are more effective in reducing duration of otorrhoea, recurrence of $A O M$ and have less side effects ${ }^{18}$. This study also showed topical antibiotics are cost-effective ${ }^{19}$. However, further research is needed in children with AOMd without grommets, since the opening to the middle ear may be smaller and the tympanic membrane heals quickly, which could prevent the drops reaching the middle ear. If topical and delayed antibiotics are shown to be non-inferior, we also need to 
understand the acceptability of such treatment to clinicians and parents and how to address any barriers to implementation.

We will address this evidence gap by assessing the clinical effectiveness and economic implications of immediate topical vs. delayed oral antibiotics and testing the hypotheses : (i) immediate antibiotics are better than placebo/no treatment for AOMd symptoms ${ }^{4}$ and (ii) delayed oral antibiotics are similar to immediate oral antibiotics in children with AOM (though with reduced antibiotic consumption) ${ }^{1}$.

The REST study is a three-arm randomised controlled trial (RCT) investigating the clinical effectiveness and economic implications of topical or delayed antibiotics compared with immediate oral antibiotics, powered for the duration and severity of the symptoms most important to parents, while also investigating adverse events, complications and $\mathrm{AOM} / \mathrm{AOMd}$ recurrence. By testing two interventions that could reduce systemic antibiotic exposure (immediate topical and delayed oral antibiotics), this study is at the forefront of research to improve antimicrobial stewardship in AOMd

\section{Methods/design}

\section{Aims and Objectives}

The key aim of this research is to investigate the clinical effectiveness and economic impact of immediate topical or delayed oral antibiotics compared with immediate oral antibiotics for symptom duration in children presenting to primary care with acute otitis media (AOM) with discharge (AOMd).

Secondary objectives are to:

- estimate the short-term cost-implications of immediate topical or delayed oral antibiotics compared with immediate oral antibiotics from the perspective of the NHS

- compare effects on duration of 'moderately bad or worse' symptoms; parent/legal guardian satisfaction with treatment; and adverse events

- compare hearing loss and rate of recurrence of AOM/AOMd at 3 months

- understand parent/ legal guardian and clinician views of participating in a trial about AOMd, adherence and satisfaction with allocated treatment.

- evaluate the impact of treatment on carriage of antibiotic resistance in the gut

\section{Trial design}

This is a multi-site, pragmatic, three-arm, individually randomised (stratified by age $<2$ vs. $\geq 2$ years), noninferiority, open trial. We compare (i) immediate topical ciprofloxacin $0.3 \%$ drops with (ii) delayed oral antibiotics; or (iii) immediate oral antibiotic in children aged 12 months to 16 years with unilateral AOMd as the presenting symptom of recent ( $\leq 7$ days) onset AOM. The primary endpoint is collected by questionnaire at day 14 post recruitment. Secondary outcomes are collected both at 14 days and at 3 month by questionnaire. 
This study is classified as a Type A study (low risk) by the Medicines and Healthcare Regulatory Agency (MHRA), with regulatory approval obtained on $4^{\text {th }}$ May 2018.

The trial design includes an internal pilot recruitment phase of 6 months duration, primarily to verify that recruitment was possible before progression to the main phase of the trial.

The REST study utilises an integrated electronic trial management platform, TRANSFoRM, that was initially developed as part of the EU FP7 TRANSFoRm project (2009-2015) and evaluated in a 60-site clinical trial in Poland ${ }^{20}$. The system integrates as a plugin within the host Electronic Health Record system through the provider's Application Programmer's Interface (API). The key features of the system include:

- Automated eligibility checking; the TRANSFoRm plugin allows the Electronic Health Record opened during a consultation to be automatically checked against the REST eligibility criteria

- Consent - the TRANSFoRm platform allows the clinician to print the study consent form for the participant to sign a record of consent can then be enter onto the platform initiating the trial's workflow.

- Integrated Randomisation system for immediate randomisation of participants during consultation,

- Trial specific Electronic Case Report Forms (eCRFs) that are presented to clinicians at appropriate appointments to complete. Some trial data is automatically retrieved from the SystmOne health record and used to part-fill the trial eCRF, which can be amended by the user.

Upon submission of the eCRF the TRANSFoRm platform automatically records a record of trial activity in the participants health record. The study flow diagram is provided in Figure 1 and the Standard Protocol Items: Recommendations for Interventional Trials (SPIRIT) Checklist in Additional file 1.

\section{Setting}

We aim to recruit 175 System One GP practices from all 15 UK CRN's. CRNs invite research active System One GP practices to express an interest in taking part in the study

\section{GP practices set-up}

Interested practices are sent a local document pack by the study team and practices are asked to return a set of documents including CV, GCP certificates and a signed site contract. Sites undergo remote training in trial conduct and the use of the TRANSFoRm electronic platform.

A site is only greenlighted once all local documents have been completed and the Principal investigator (PI) has completed the online training. At this point the TRANSFoRm electronic platform is installed onto the PI' computer. Once a site is greenlighted additional clinicians from that practice can complete the REST training package, be added to the site delegation log and the TRANSFoRm platform can be downloaded onto additional practice computers. 


\section{Participants and eligibility}

Eligible participants are children aged $\geq 12$ months to $<16$ years whose parents/legal guardians are seeking primary medical care for acute onset unilateral otorrhoea as the presenting symptom of recent ( $\leq 7$ days) onset AOM. Detailed inclusion and exclusion criteria are detailed in table 1.

\section{Patient screening and recruitment}

The process of patient screening and recruitment is detailed below:

1. Children aged aged $\geq 12$ months to $<16$ years accompanied by a parent/ legal guardian present to their GP with suspected AOMd

2. GP invites parent and child to participate in the REST study and provides a Parent information leaflet (Child information leaflet for children above 6 years old)

3. GP seeks verbal agreement from parents and assesses the child for eligibility using the TRANSFoRm electronic platform.

4. Informed consent/assent is sought from parents of eligible children, baselines data and contact details are collected via the TRANSFoRm platform

5. Child is randomised using the TRANSFoRm platform and if allocated the drops or immediate antibiotics an FP10 prescription (Standard UK prescription) is issued.

Table 1: Inclusion and Exclusion Criteria 
Patient Selection Criteria

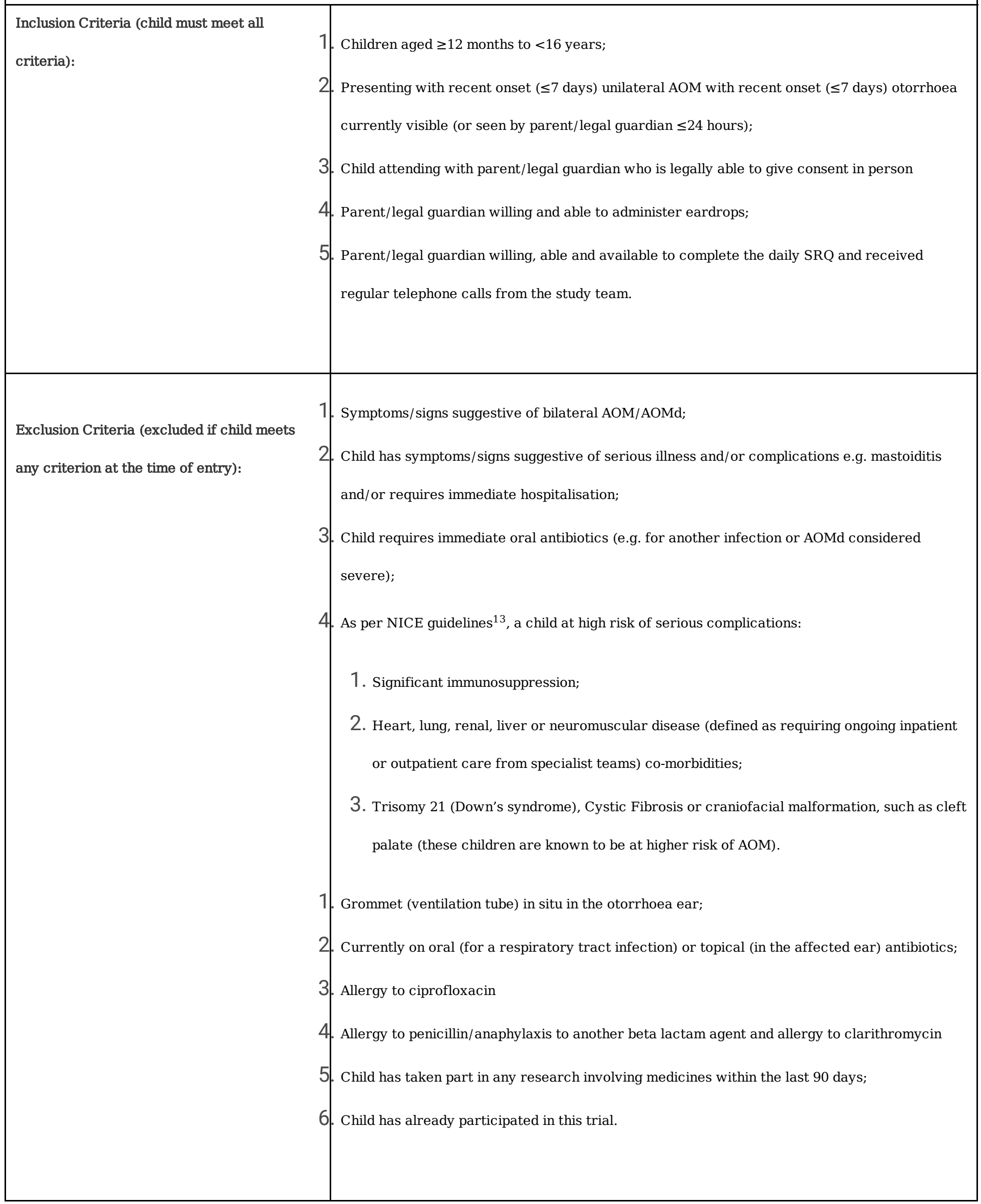




\section{Randomisation}

After confirming eligibility and obtaining informed consent, participants will be randomised (stratified by age) to either (i) immediate topical ciprofloxacin $0.3 \%$ drops with (ii) delayed oral antibiotics; or (iii) immediate oral antibiotics.

The randomisation sequence is generated by the Bristol Randomised Trials Collaboration (BRTC) and supplied to the TRANSFoRm electronic platform to be allocated to each successive participant recruited. A system for checking the correct randomisation allocation is built in to the TRANSFoRm platform. Clinicians will not be able to determine treatment allocation pre-randomisation.

\section{Consent}

Parent Information Sheets (Additional file 2) will be given to the parents of potentially eligible children and discussed before consent is sought. Informed consent will be obtained from the parent or legal guardian of each child. Assent will be obtained from all children over the age of 6 . Parents will also be given be the opportunity to consent to stool sample collections, declining to consent to this element of the study will not exclude participation from other elements of the study.

\section{Withdrawal}

Participants remain in the trial unless they choose to withdraw, or if they are unable to continue. Parents can choose to completely withdraw their child full or withdraw from specific elements of the study without giving a reason. Any data collected up until this point will be retained for analysis. Information of the withdrawal criteria are detailed in the parent information leaflet

\section{Interventions}

\section{Choice of comparator}

We selected ciprofloxacin $0.3 \%$ drops as our topical antibiotic because it:

- is active against all common otopathogens ${ }^{3}$;

- is non-ototoxic;

- is widely and routinely available in the UK;

- is colourless so will not interfere with assessing otorrhoea;

- will provide complementary evidence to the ZonMw funded trial, which is using an antibiotic-steroid combination.

We decided to avoid aminoglycoside drops because of concerns about potential ototoxicity. We have proposed delayed oral antibiotics as the second intervention since our previous trials $1,21,22$ have achieved 
significant reductions in oral antibiotic consumption compared with immediate antibiotic prescribing (and similar symptom relief). Immediate oral amoxicillin (clarithromycin if allergic to penicillin) is the comparator as it reflects usual care and is well tolerated.

\section{Intervention description}

Arm 1: (control) current usual care - oral amoxicillin suspension three times daily for 7 days (or oral clarithromycin twice daily for 7 days if allergic to penicillin).

Arm 2: (intervention) antibiotic drops, to be instilled three times daily into the discharging ear. Parents will be given written advice regarding how to administer the drops. This will include: (i) cleaning the outer ear of discharge that can be easily removed with a tissue; (ii) tilting the child's head to one side (to approximately 90 degrees) when applying the eardrops; and (iii) maintaining the tilt for a few minutes to improve penetration of the drops.

Arm 3:(intervention) a 'delayed' prescription for oral amoxicillin suspension antibiotics three times daily for 7 days (or oral clarithromycin twice daily for 7 days if allergic to penicillin). Parents will be given written advice to delay the prescription will consist of: (i) advising the prescription is only 'dispensed' at a pharmacy if symptoms worsen or are not starting to improve by 4 days; and (ii) safety - netting advice regarding the symptoms that should prompt review consultation (increasing pain, high temperatures, headaches, irritability or reduced feeding).

All groups will also receive standard advice to complete the antibiotic course and how to managepain, fever and other symptoms (e.g. use of paracetamol/ibuprofen).

\section{Post-trial care}

Following participation in the study, children are returned to usual care by their GP. All participants will receive a summary of the results of the trial.

\section{Outcome measures}

The primary outcome measure is time to resolution of the following symptoms: pain, fever, being unwell, sleep disturbance, otorrhea and episodes of distress. The primary outcome is the time until all symptoms are rated by parents as "no" or "very slight" problem. This will be recorded by parents in the Symptom Recovery Questionnaire (SRQ).

Secondary outcome measures:

- duration of 'moderately bad or worse' symptoms (pain, fever, being unwell, sleep disturbance, otorrhoea; episodes of distress/crying;

- appetite and interference with normal activities up to 14 days;

- antibiotic and analgesic use; 
- adverse events - diarrhoea, rash, vomiting, serious complications;

- treatment adherence;

- parent/ legal guardian satisfaction with treatment;

- NHS resource use at 14 days;

- repeat $\mathrm{AOM}$ and AOMd episodes, serious complications and OM6 hearing questionnaire at 3 months;

- qualitative evaluation of recruitment, medication satisfaction, adherence and follow up.

- analysis of stool sample to assess burden of resistance

\section{Assessment and follow up}

The components and timing of follow-up measures are shown in Fig 2.

\section{Clinician recorded outcomes: Baseline data collection form and contact form}

During the consultation, clinicians will complete a baseline data collection form and contact detail form for all eligible participants. The baseline data collected includes acute clinical data and any relevant medical history relating to AOMd incidence.

A contact details form will record information from parent/legal guardian including name, address. telephone number and availability to take calls from the research nurse.

\section{Patient reported outcomes}

All parents/legal guardians are asked to complete a daily SRQ recording the symptoms identified by parents/legal guardians as important. The SRQ will be provided in electronic format via the TRANSFoRm app or in a paper version. The SRQ will provide a daily record of symptom burden and will be completed up to 14 days. The primary outcome will be collected using the SRQ with research nurse telephone calls on day $1,3,7,10$ and 14 .

On day 7 and day 14 telephone calls, information on the use of healthcare resources including information about primary care contacts, use of 111 and walk-in centres, and hospital services will be obtained.

At month 3, use of hospital services will be collected by review of the patients' EHR.

The final questionnaire will be sent three months after randomisation either electronically (web or iOS/Android app) via the TRANSFoRm platform or paper questionnaire. The questionnaire will ask parent/legal guardian-reported hearing loss at 3 months measured using the $0 \mathrm{M}^{23}$ questionnaire.

\section{Storage and analysis of microbiological clinical samples}

Plans for the collection, storage and evaluation of biological specimens can be found in Additional file 3 . 


\section{Economic data collection}

The economic evaluation will explore the relationship between cost and outcomes for the three proposed methods of treating otitis media with discharge. This will be done for the short term ( 14 days) to assess the cost-effectiveness of immediate topical or delayed oral antibiotics compared with immediate oral antibiotics, and the longer term (3 months) in order to capture the effects of any recurrence of symptoms and lasting side effects. The perspectives will be from the NHS, parents/carers, and lost productivity due to time off work and school.

During the trial, participants will complete a symptom diary which will record any use of healthcare resources not available from the GP notes (for example, community care and the use of 111 and walk-in centres) and non-healthcare costs incurred by parents/carers during the first 14 days such as travel costs, purchase of over-the-counter medications, childcare, and loss of earnings due to time off work. We will also ask about time off nursery/school.

\section{Qualitative data collection}

The qualitative evaluation will explore the views and experiences of the trial processes, the acceptability of the different treatment options, and the barriers and facilitators to their use within, and future uptake outside the trial.

Purposive sampling ${ }^{24}$ will select participants in order to capture maximum variation in views and experiences of a range of parents and primary care professionals. From parents that agree to trial participation and the interview, a purposive sample will be drawn in relation to site, arm of the trial, and socio-demographic variables such as socio-economic status. Parents who decline trial participation will also be invited to be interviewed. Primary care professionals involved in trial processes will also be purposively sampled in relation to site. Sample sizes will be determined by data saturation ${ }^{24}$, such that no new themes are emerging from the data by the end of data collection. We anticipate including up to 20 clinicians, 20 participant parent interviews and 15 parent decliner/withdrawal telephone interviews.

In-depth interviews will be conducted with participating parents (from all arms of the trial) 14 days after randomisation ${ }^{25}$. Interviews with parents who declined to participate will be conducted within seven days of declining. These will be conducted by telephone at a time of the participant's choosing. Interviews with primary care professionals will be conducted after 3-6 months of involvement in the trial to try to capture those with experience of recruitment. A flexible topic guide will be devised to ensure that the primary issues are covered across all interviews, but it will incorporate considerable flexibility to enable participants to introduce unanticipated issues, and they will be modified to reflect findings as they emerge. The researcher will use open-ended questioning techniques to elicit participants' experiences and views of key events and participants will be asked to provide examples. Primary care professional's interviews are expected to last around 30-45 minutes, parent interviews 30-40 minutes and those with parents who decline trial participation 10-20 minutes. Interviews will be recorded using a digital voice recorder, transcribed and anonymised to protect confidentiality. 


\section{Trial oversight}

The study is overseen by a Trial Management Group that meet on a monthly basis and consist of the $\mathrm{Cl}$, grant holders, study sponsor and any other staff responsible for the delivery of the trial. The Trial steering committee (TSC) provide independent supervision of the trial and oversees trial progress. The TSC consists of an independent chair and three other independent members including a clinical trial list, statistician, PPI representative and the $\mathrm{Cl}$. The Data monitoring committee (DMC) monitors patient safety and trial data efficacy and consists of an independent chair, three other independent members and the $\mathrm{Cl}$.

All serious adverse events (SAE'S) are recorded and notified as appropriate to the relevant authorities.

\section{PPI}

A comprehensive programme of PPI engagement was conducted during the set-up stage to inform the development of the symptom diary data collection, of the parent and child-facing trial documentation. PPI contributors attend both the TMG and TSC meetings, providing ongoing guidance. PPI members will help identify nonacademic dissemination avenues, and will advise on materials for press releases, print media, social media and parent facing materials, including presentation of results using a parent/child friendly animation

\section{Data management and confidentiality}

Study data are collected and stored using the TRANSFoRm ${ }^{20} \mathrm{GCP}$-validated clinical trial platform that is integrated into the GP's Electronic Health Record system. Once a patient presents to the GP with one of the specified otitis media disease codes, eligibility screening is run in the background on their EHR. Should the patient be found suitable for inclusion in the study, the GP is asked to consent the patient/legal guardian. No data is captured in TRANSFoRm until the parent/legal guardian has consented to theirs and their child's participation. The data is captured through electronic Case Report Forms (eCRFs) completed through the EHR system, and the Patient Reported Outcome Measures (PROMs) completed at set timepoints by the patient through a web portal on their mobile devices, tablets, or desktop computers at home. The data is transmitted using a secure connection and stored inside an encrypted database hosted at King's College London. Subset of participant information is additionally stored in the REDCap database hosted at the University of Bristol, added by a member of the REST study team.

Both the TRANSFoRm electronic platform and REDCap incorporate data entry and validation rules to reduce data-entry errors and double data entry. Trial staff will ensure that the participants' anonymity is maintained through protective and secure handling and storage of patient information at the trial centre's. Data will be anonymised as soon as it is practical to do so in line with the Data Protection Act 1998. Participant's data is securely held on the databases in line with data protection legislation. 
To comply with the fifth Principle of the Data Protection Act 1998 (this process will be reviewed and updated accordingly with any updates to the guidelines), personal data will not be kept for longer than is required for the purpose for which it has been acquired. Data will be held in compliance with the sponsor's standard operating procedures.

\section{Sample size}

Our previous trial compared immediate with delayed antibiotics. ${ }^{1}$. Children with AOMd took a median of 3 days (IQR 2,4$)$ to achieve the REST primary outcome. Our PPI advised a 1.25 day non-inferiority margin (equivalent to an absolute difference in cure rate of $19.5 \%$ at 3 days). A two-group non-inferiority trial normally assumes $2.5 \%$ one-sided Type I error. Using $1.25 \%$ Type I error to detect non-inferiority for two comparisons with $90 \%$ power, complete outcome data needed for 106 per arm: 399 with $20 \%$ attrition.

\section{Statistical analysis}

A flow of participants through the trial will be summarised in a CONSORT diagram. Descriptive statistics of baseline clinical and socio-demographic characteristics will be presented to describe the study sample and to ascertain comparability of the randomisation groups.

Data from the internal pilot phase of the study will be assessed against predefined/pre-agreed stop/go criteria to inform the decision as to whether to continue the trial to 'main' phase. The proposed 'traffic light' (stop/go) criteria are based on descriptive statistics summarising recruitment, retention and adherence.

The primary analysis will be carried out under the intention-to-treat (ITT) principle, analysing participants as randomised without the imputation of missing data. Kaplan-Meier survival curves will be plotted to depict the probability of symptom resolution over time. Symptom resolution over the 14 days of follow-up will be compared between children allocated to immediate oral antibiotics and those allocated to each of the other treatment groups using a Cox proportional hazards regression model, adjusted for age (stratification variable). The primary outcome will also be analysed using an Accelerated Failure Time (AFT) model, which has previously been recommended for studies of resolution of infectious diseases as previous research has suggested that symptoms of AOM will be resolved in $90 \%$ of children by day 8 .

The proportion of participants in the immediate topical and delayed oral antibiotics arms who achieve symptom resolution within 3 days will be compared (separately) to those in the immediate oral antibiotics arm. The absolute difference will be calculated and reported alongside the associated confidence interval, it will then be reported whether or not the lower limit of the confidence interval lies within the maximum unimportant difference.

Analysis of secondary outcomes will utilise regression models appropriate for the nature of the outcome measure (i.e. logistic regression for binary outcomes, poisson on negative binomial regression for count data). 
The primary analysis model will be repeated but with the outcome of symptom resolution being defined as when all symptoms are rated as being "normal/none", "very slight problem" or "slight problem" (compared to the primary outcome of symptom resolution being defined as all symptoms being rated as "normal/none" or "very slight problem"). The primary analysis will also be repeated under the per-protocol approach (rather than ITT). The sensitivity of the primary analysis to the impact of missing data will also be explored by repeating the analysis after the imputation of missing primary outcome data.

\section{Economic data analysis}

The primary economic evaluation will explore the relationship between cost and outcome for the three treatments for AOMd (immediate topical, delayed oral and immediate oral antibiotics) from an NHS perspective at 14 days post-randomisation. This will take the form of a simple comparison of NHS costs and outcomes over a period of two weeks from randomisation.

A secondary cost analysis will evaluate the difference in NHS secondary care costs between the trial arms for the three months following randomisation.

All resources will be valued using unit costs from established sources. These will include Unit Costs of Health and Social Care ${ }^{26}$ for primary and community care, NHS Reference Costs ${ }^{27}$ for hospital care and the $\mathrm{BNFC}^{28}$ for prescribed medication.

Differences in NHS resources and costs between the arms will be analysed initially using Ordinary Least Squares (OLS) regression. The distribution of residuals from the regression models will then be examined and a decision will be made as to whether OLS is appropriate or another type of regression model should be considered (e.g. Generalised Linear Models (GLM)).

A cost consequence analysis will then be conducted in which the costs to the NHS of the three treatments at 14 days post-randomisation will be compared with the primary clinical outcome. Areas of uncertainty in assumptions will be subjected to sensitivity analyses to test the robustness of the results.

\section{Qualitative data analysis}

Interview transcripts will be imported into NVIVO 12 qualitative data analysis software. Analysis will begin shortly after data collection starts and will be ongoing and iterative - informing further data collection and identifying changes needed to the topic guide. Thematic analysis ${ }^{29}$, utilising a data-driven inductive approach, will be used to identify and analyse patterns and themes of particular salience for

participants and across the dataset using constant comparison techniques ${ }^{30,31}$. A subset of transcripts will be independently double coded by members of the team ( $\mathrm{CC}$ and $\mathrm{JH})$; any discrepancies will be discussed within the team and resolved to achieve coding consensus and maximal rigour.

\section{Blinding}


Once the allocation is revealed, neither clinicians nor the child participant or their parent/legal guardian will remain blind to their allocated treatment. Codes will be assigned to the database, which will preserve blinding of study personnel. The senior statistician will remain blind to knowledge of which treatment is represented by each treatment code until final results have been shared with the Data Monitoring Committee (DMC).

\section{Dissemination}

We will publish the trial results in peer-reviewed journals and present at national and international meetings. With the assistance of our collaborators and PPI we will disseminate the study findings to an international audience. All participants will be offered a lay summary of the main findings of the study.

\section{Discussion}

This article outlines a pragmatic, three-arm, individually randomised, trial, which aims to recruit from 175 GP practices across the UK. The aim is to establish evidence for the non-inferiority of: (i) Immediate topical ear drops, ciprofloxacin $0.3 \%$; (ii) Delayed oral amoxicillin (clarithromycin); or (iii) Immediate oral amoxicillin (clarithromycin). The non-inferiority will be based on time to resolution of all significant symptoms. Secondary outcomes will include cost-effectiveness, duration of moderately bad or worse symptoms and repeat AOMd episodes.

Recruitment of 399 children with AOMd over a 22 month period presented challenges around the design of the REST study. Only 2-3 patients children were expected to presentation at each GP practice over the 22 month recruitment period requiring at least $175 \mathrm{GP}$ practices to take part in the study.

In order to make this study feasible we needed to consider novel and streamline mechanism to facilitate greenlighting of sites, training of site staff, identification of eligible participants and the collection of highquality data.

We employed several different strategies in order to address these elements of the study, these included:

- Development of a remote training platform to deliver REST study training to GP's and staff at recruiting practices

- A light touch Green Light Process (GLP), maximising the number of staff at the GP practices able to recruit to the REST study by providing quick and accessible study specific training

We used the TRANSFoRm electronic platform, (integrates into the health records) to automatically create a pop-up alert to the clinician when an eligible patient presents. The system automatically checked the participants eligibility and part filled the study eCRFs reducing the efforts of the clinician to record study data.

\section{Trial Status:}


Currently Fifty System One GP practices have been greenlighted across 7 CRN regions in the UK. The first child was recruited to the study on 5th April 2019 with recruitment currently ongoing. A total of 22 children were recruited to the REST study, with recruitment being closed on 31st May 2020.

\section{Protocol}

Version 7.0, 31.10.2018

Open to recruitment

7th April 2019

Planned recruitment closure date

31 st March 2020

\section{Abbreviations}

AOMd

Acute Otitis Media with Discharge

AE

Adverse Event

$\mathrm{Cl}$

Chief Investigator

CRF

Case Report Form

EHR

Electronic Health Record

GP

General Practitioner

$\mathrm{NHS}$

National Health Service

NICE

National Institute of Health and Social Care

PI

Principal Investigator

TMG

Trial Management Group

TSC

Trial Steering Committee

DMC

Data Monitoring Committee

\section{Declarations}

\section{Acknowledgements}


This study was designed and delivered in collaboration with the Bristol Randomised Trials Collaboration (BRTC), part of the Bristol Trials Centre. The University of Bristol is acting as sponsor for this trial and the NHS Bristol, North Somerset and South Gloucestershire Group (CCG) host the trial. The authors would like to thank all of the participants, principle investigators and their teams at each of the REST study sites for their involvement. The authors would like to thank all 15 UK CRN's for their role in the study. The authors would also like to thank members of their TSC and DMC.

\section{Authors' contributions}

Alastair D Hay and Michael Moore are the Co-Chief Investigators of REST; they conceived the study, participated in its design and coordination and helped to draft the manuscript. Kathryn Curtis developed the trial procedures, manuscript and managed the coordination of the study. Jodi Taylor also assisted with the coordination of the study and drafting the manuscript. Vibhore Prasad contributed to the study design and manuscript. Anne Schilder and Scott Wilkes also assisted with the study design. Vasa Curcin developed the TRANSFoRm electronic trials platform used in the study. Nicholas Turner and Richard Morris designed the statistical analysis, Christie Cabral and Jeremy Horwood developed the qualitative elements of the study.

All authors contribute to the oversight of the study via the TMG, read and commented on manuscript drafts and approved the final manuscript

\section{Funding}

This research is funded by the National Institute for Health Research (NIHR) Health Technology Assessment (HTA) programme (funder ref: 16/85/01). See Additional File 4 for the agree to fund letter. The views expressed in this publication are those of the authors and not necessarily those of the NHS, the NIHR or the Department of Health and Social Care.

\section{Availability of data and materials}

Not applicable

\section{Ethics approval and consent to participate}

The research protocol was given a favorable opinion by the South Central -Oxford B Research Ethics Committee on 22 May 2018. This ethics approvals applies to all NHS sites taking part in the REST study. Parents/Legal guardians will be asked for their permission for their child to participate in the study and will be asked to provide their written, informed consent prior to enrollment. Any amendments to the protocol will be reported accordingly to the regulatory bodies (See Additional File 5 for the confirmation of favourable opinion by the Ethics Committee letter).

\section{Consent for publication}

Not applicable 


\section{Competing interests}

The authors declare that they have no competing interests.

\section{References}

1. Little P, Gould C, Williamson I, et al. Pragmatic randomised controlled trial of two prescribing strategies for childhood acute otitis media. BMJ 2001;322(7282):336-42.

2. Watson L, Little P, Moore $M$, et al. Validation study of a diary for use in acute lower respiratory tract infection. Family Practice 2001;18(0263-2136):553-54.

3. Smith L, Ewings P, Smith C, et al. Ear discharge in children presenting with acute otitis media: observational study from UK general practice. British Journal of General Practice 2010;60(571):10105. doi: DOI 10.3399/bjgp10X483148

4. Rovers MM, Glasziou P, Appelman CL, et al. Antibiotics for acute otitis media: a meta-analysis with individual patient data. Lancet 2006;368(9545):1429-35.

5. Hollinghurst S, Gorst C, Fahey T, et al. Measuring the financial burden of acute cough in pre-school children: a cost of illness study. BMC Family Practice 2008;9(1):10. doi: Doi 10.1186/1471-2296-9-10

6. Hollinghurst $S$, Redmond NM, Costelloe $C$, et al. Paracetamol plus ibuprofen for the treatment of fever in children (PITCH): economic evaluation of the randomised controlled trial. $B M J$ 2008;337(7672):a1490. doi: Doi 10.1136/Bmj.A1490

7. Wolleswinkel-van den Bosch JH, Stolk EA, Francois M, et al. The health care burden and societal impact of acute otitis media in seven European countries: Results of an Internet survey. Vaccine 2010;28:G39-G52. doi: DOI 10.1016/j.vaccine.2010.06.014

8. Hay AD, Heron J, Ness A, et al. The prevalence of symptoms and consultations in pre-school children in the Avon Longitudinal Study of Parents and Children (ALSPAC): a prospective cohort study. Family Practice 2005;22(4):367-74. doi: DOI 10.1093/fampra/cmi-35

9. Williamson I, Benge S, Mullee $\mathrm{M}$, et al. Consultations for middle ear disease, antibiotic prescribing and risk factors for reattendance: a case-linked cohort study. Br J Gen Pract 2006;56(524):170-75.

10. Finkelstein JA, Metlay JP, Davis RL, et al. Antimicrobial use in defined populations of infants and young children. Arch Pediatr Adolesc Med 2000;154(4):395-400.

11. Ashworth M, Charlton J, Ballard K, et al. Variations in antibiotic prescribing and consultation rates for acute respiratory infection in UK practices 1995-2000. Br J Gen Pract 2005;55:603-08.

12. Ashworth M, Cox K, Latinovic $R$, et al. Why has antibiotic prescribing for respiratory illness declined in primary care? A longitudinal study using the General Practice Research Database. Journal of Public Health 2004;26(3):268-74.

13. NICE. Respiratory tract infections: prescribing of antibiotics for self-limiting respiratory tract infections in adults and children in primary care, 2008. 
14. Foliaki S, Pearce N, Bjorksten B, et al. Antibiotic use in infancy and symptoms of asthma, rhinoconjunctivitis, and eczema in children 6 and 7 years old: International Study of Asthma and Allergies in Childhood Phase III. J Allergy Clin Immunol2009;124(5):982-9. doi: 10.1016/j.jaci.2009.08.017

15. Goossens $H$, Ferech $M$, van der Stichel R, et al. Outpatient antibiotic use in Europe and association with resistance: a cross-national database study. Lancet 2005;365:579-87.

16. Costelloe $C$, Metcalfe $C$, Lovering $A$, et al. Effect of antibiotic prescribing in primary care on antimicrobial resistance in individual patients: systematic review and meta-analysis. $B M J$ 2010;340(May18_2):c2096. doi: Doi 10.1136/Bmj.C2096

17. Department of Health. UK Antimicrobial Resistance Strategy and Action Plan. London, 2000.

18. van Dongen TMA, van der Heijden GJMG, Venekamp RP, et al. A Trial of Treatment for Acute Otorrhea in Children with Tympanostomy Tubes. New England Journal of Medicine 2014;370(8):723-33. doi: Doi 10.1056/Nejmoa1301630

19. van Dongen TM, Schilder AG, Venekamp RP, et al. Cost-effectiveness of treatment of acute otorrhea in children with tympanostomy tubes. Pediatrics 2015;135(5):e1182-9. doi: 10.1542/peds.2014-3141

20. Delaney BC, Curcin V, Andreasson A, et al. Translational Medicine and Patient Safety in Europe: TRANSFoRm-Architecture for the Learning Health System in Europe. Biomed Res Int 2015;2015:961526. doi: 10.1155/2015/961526

21. Little P, Rumsby K, Kelly J, et al. Information Leaflet and Antibiotic Prescribing Strategies for Acute Lower Respiratory Tract Infection: A Randomized Controlled Trial. JAMA 2005;293(24):3029-35.

22. Little $P$, Williamson I, Warner $G$, et al. Open randomised trial of prescribing strategies in managing sore throat. British Medical Journal 1997;314(7082):722-27.

23. Kubba H, Swan IRC, Gatehouse S. How appropriate is the $0 M 6$ as a discriminative instrument in children with otitis media? Archives of Otolaryngology-Head \& Neck Surgery 2004;130(6):705-09. doi: DOI 10.1001/archotol.130.6.705

24. Sandelowski, M. (1995) Sample size in qualitative research. Research in Nursing and Health, 18:179-83

25. Britten N. (1995) Qualitative interviews in medical research. BMJ; 22; 311:251-3

26. Curtis L, Burns A. Unit Costs of Health and Social Care 2015. PSSRU University of Kent 2015:284.

27. Department of Health. NHS Reference costs 2014

28. Royal Pharmaceutical Society of Great Britain. British National Formulary for Children. 2016

29. Braun V, Clarke V. (2006) Using thematic analysis in psychology. Qualitative Research in Psychology;3:77-101

30. Boyatzis, R. Transforming qualitative information: Thematic analysis and code development. (1998). Thousand Oaks, CA: Sage

31. Charmay K. (2006) Constructing grounded theory: a practical guide through qualitative analysis. London: Sage 


\section{Additional Files}

- Additional file 1: REST Standard Protocol Items: Recommendations for Interventional Trials (SPIRIT) Checklist (PDF)

- Additonal File 2: The Runny Ear Study Parent Information Sheet (PDF)

- Additional File 3: REST study microbiology protocol (PDF)

- Additonal File 4: Agree to fund letter from NIHR HTA (PDF)

- Additional File 5: Confirmation of Favourable opinion by Ethics Commitee Letter (PDF)

\section{Figures}


National study awareness raising activities:

- Study team to write to larger and research active GP practices nationally via the NIHR Coordinating Centre

- $\frac{1}{2}$ Support from the NIHR CRN primary care lead (hub-and-spoke model) Interested clinicians (GPS and Nurses) do online study and GCP training with online verification of completed training

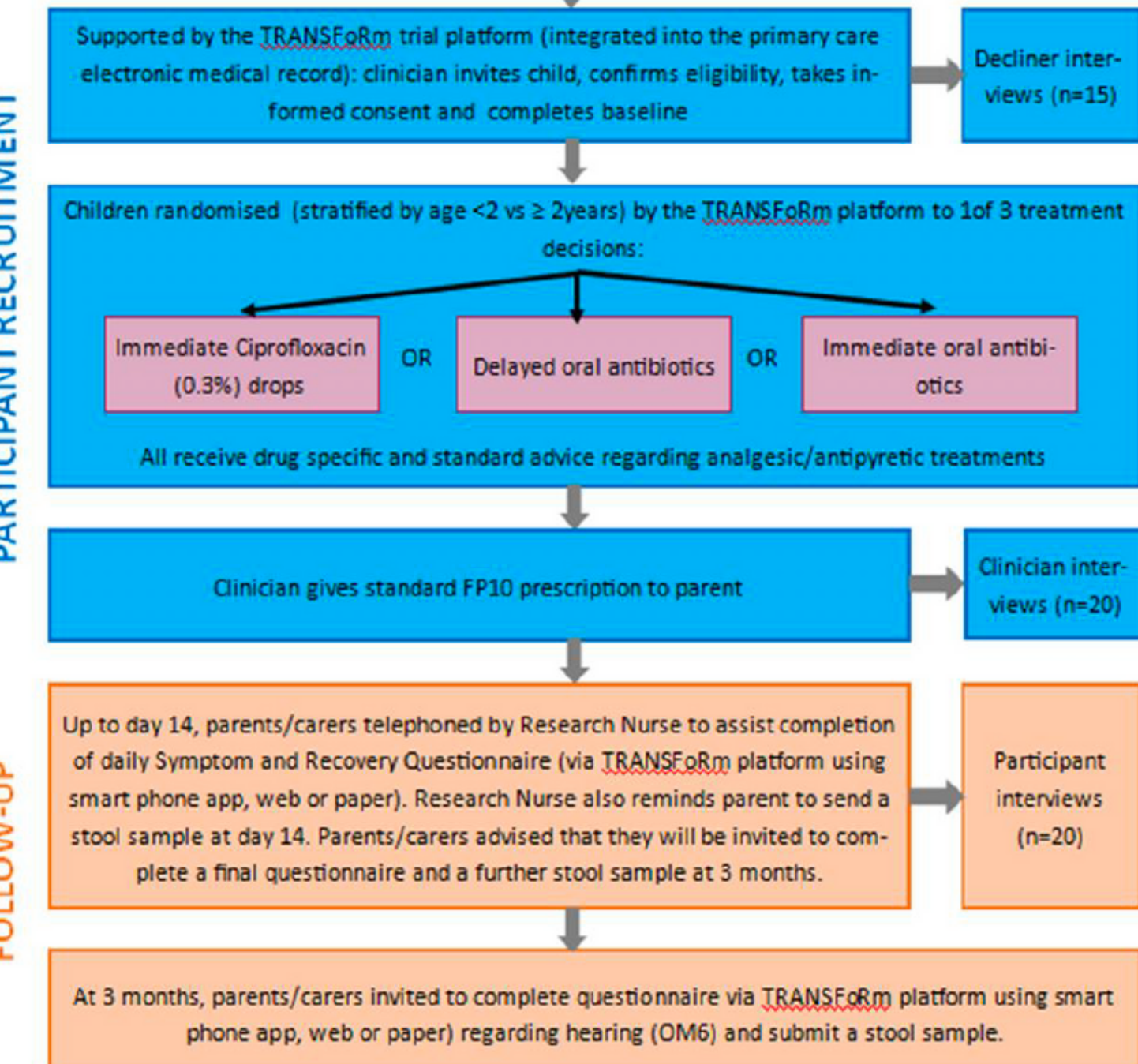

\section{Figure 1}

Trial Flow Chart 


\begin{tabular}{|c|c|c|c|c|c|c|c|c|c|}
\hline \multicolumn{10}{|c|}{ Study Period } \\
\hline \multirow[b]{2}{*}{ Timepoint } & \multirow{2}{*}{$\begin{array}{l}\text { Screening } \\
\text { Pre } \\
\text { consent }\end{array}$} & \multirow{2}{*}{$\begin{array}{l}\begin{array}{l}\text { Patient } \\
\text { enrolment }\end{array} \\
\text { Baseline }\end{array}$} & \multicolumn{7}{|c|}{ Post enrolment } \\
\hline & & & $\begin{array}{l}\text { Day } \\
1 \\
\end{array}$ & $\begin{array}{l}\text { Day } \\
3\end{array}$ & $\begin{array}{l}\text { Day } \\
7\end{array}$ & $\begin{array}{l}\text { Day } \\
10 \\
\end{array}$ & \begin{tabular}{|l|} 
Day \\
14 \\
\end{tabular} & $\begin{array}{l}6 \\
\text { weeks }\end{array}$ & $\begin{array}{l}3 \\
\text { months }\end{array}$ \\
\hline $\begin{array}{l}\text { Pre-eligibility } \\
\text { screening }\end{array}$ & $\mathrm{x}$ & & & & & & & & \\
\hline $\begin{array}{l}\text { Eligibility } \\
\text { confirmation }\end{array}$ & $\mathrm{x}$ & & & & & & & & \\
\hline $\begin{array}{l}\text { Baseline Data } \\
\text { Collection Form }\end{array}$ & & $x$ & & & & & & & \\
\hline $\begin{array}{l}\text { Informed } \\
\text { Consent }\end{array}$ & & $x$ & & & & & & & \\
\hline Randomisation & & $x$ & & & & & & & \\
\hline $\begin{array}{l}\text { Contact Details } \\
\text { form }\end{array}$ & & $\mathrm{x}$ & & & & & & & \\
\hline \multicolumn{10}{|l|}{ Interventions } \\
\hline \multicolumn{10}{|l|}{$\begin{array}{l}\text { Immediate Oral } \\
\text { Antibiotics }\end{array}$} \\
\hline \multicolumn{10}{|l|}{$\begin{array}{l}\text { Delayed Oral } \\
\text { Antibiotics }\end{array}$} \\
\hline \multicolumn{10}{|l|}{$\begin{array}{l}\text { Ciprofloxacin } \\
\text { ear drops }\end{array}$} \\
\hline \multicolumn{10}{|l|}{ Assessments } \\
\hline \multicolumn{10}{|l|}{$\begin{array}{l}\text { Symptom } \\
\text { questionnaire }\end{array}$} \\
\hline $\begin{array}{l}\text { Research Nurse } \\
\text { telephone calls }\end{array}$ & & & $x$ & $\mathrm{X}$ & $x$ & $x$ & $x$ & & \\
\hline $\begin{array}{l}6 \text { wk contact } \\
\text { for pts w/ } \\
\text { symptoms post } \\
\text { D14 }\end{array}$ & & & & & & & & $x$ & \\
\hline $\begin{array}{l}\text { OM6 } \\
\text { questionnaire } \\
\text { 3mth QoL }\end{array}$ & & & & & & & & & $x$ \\
\hline $\begin{array}{l}3 \text { month Notes } \\
\text { Review }\end{array}$ & & & & & & & & & $\mathrm{x}$ \\
\hline \multicolumn{10}{|l|}{$\begin{array}{l}\text { Parent } \\
\text { Interviews }\end{array}$} \\
\hline \multicolumn{10}{|l|}{$\begin{array}{l}\text { Decliner } \\
\text { Interviews }\end{array}$} \\
\hline GP interviews & & & & & & & & & $\overline{ }$ \\
\hline
\end{tabular}

\section{Figure 2}

Participant recruitment and follow-up timeline. *Completed daily from day 1 - day 14

\section{Supplementary Files}

This is a list of supplementary files associated with this preprint. Click to download. 
- Additonalfile4.pdf

- Additonalfile2.pdf

- Additionalfile1.pdf

- Additonalfile5.pdf

- Additionalfile3.pdf 